\title{
El trauma de un pueblo. ¿Qué paz después de la guerra?*
}

\author{
Guy Briole**
}

\section{"El pasado, al no iluminar ya el porvenir, el espiritu camina en las tinieblas" Alexis de Tocqueville (1951, p. 336)}

Las guerras ya no son mundiales, es el mundo el que está por todas partes en guerra. Lo está sin discontinuar, recorrido por la contaminación de una onda que se quiere purificadora y salvadora, y que se propaga llevando cada vez más la marca de lo religioso, el rasgo racial, la diferencia de pertenencias. ¿Cómo, en esta deriva del mundo, no reconocer la prolongación de lo que Jacques Lacan identificaba, al salir de la guerra, en septiembre de 1945, como una disolución del sentido moral? La ignominia localizada en el corazón de la vieja Europa y el horror de Hiroshima han dejado a los hombres pasmados e incapaces de hablar del traumatismo impensable.

Se han callado para sobrevivir, satisfaciendo la necesidad de reconciliaciones aparentes, dejando el odio intacto, profundo, ardiente, al quedar contenido en sus refugios imaginarios.

En este "desconocimiento sistemático del mundo", Lacan (2001a) ha reconocido "esas mismas modalidades de defensa que el individuo utiliza en

* El siguiente escrito corresponde a la conferencia inaugural dictada por el profesor Guy Briole el 7 de septiembre de 2017 en el marco del evento Salud Mental, Trauma y Posconflicto, realizado en la Universidad del Rosario, Bogotá, Colombia, organizado por la Escuela de Medicina y Ciencias de la Salud y la Cancillería de la Universidad. Lo reproducimos a continuación como editoral de este número [Nota del Director].

Traducción de Alín Salom.

** Guy Briole, Profesor de Val-de-Grace y Secretario de la Asociación Mundial de Psicoanálisis (AMP). la neurosis contra su angustia, y con un éxito no menos ambiguo, también paradójicamente eficaz, y que sella del mismo modo, ¡ay!, un destino que se transmite a las generaciones sucesivas" (p. 101).

\section{Lo que se transmite}

En la generación de posguerra, casi todos los ciudadanos que componen un país - hombres, mujeres y niños - son supervivientes o descendientes de los que han sobrevivido a las guerras que han atravesado y devastado sus países.

En Europa, es esencial decir que para aquellos que han sido afectados por la guerra del 39-45, las marcas ya venían de antes: de la carnicería que fue 14-18 para los europeos; las masacres que culminaron entre 1915 y 1918 , para los armenios; las guerras coloniales, las guerras civiles que han conducido al establecimiento de dictaduras devastadoras para el lazo social. La posguerra no omitió, entre las consecuencias de la guerra fría, producir el odio que se infiltra en un pueblo sometido, cuando una ideología lo mantiene bajo su yugo.

Lo mismo ocurrió en América Latina, cuando dictaduras de las más feroces condujeron países al caos; y los intereses desmesurados de las fuerzas capitalistas no tuvieron ninguna contención ni vergüenza en apoyar la lucha implacable entre ciudadanos del mismo país, que genera una guerra civil.

Por todas partes en el mundo, como lo hemos subrayado, estas heridas abiertas sangran con la sangre de la locura de los hombres, de no poder renunciar a destruirse entre ellos.

Hoy en día, son los hijos e hijas, los nietos o los biznietos los que llevan estas heridas, aún abiertas 
para muchos: son supervivientes hechos de los jirones de las guerras. A pesar de que todos los que han atravesado las guerras, la tortura, la negación de ellos mismos o de su pueblo de pertenencia, hayan tenido dificultades para hablar del horror que han podido vivir psicológicamente y en su cuerpo, algo de estas historias, a pesar de todo, se ha transmitido.

No es, pues, sin daño - a veces a pesar de su obstinación en callarse o bien confrontados a esta parte imposible de comunicar- como se ha hecho una transmisión, por parte de los que han vuelto de estos infiernos y para quienes la reducción del cuerpo a lo que queda de vida sería como una enfermedad incurable. Los hijos de un superviviente habían inventado un nombre a esta enfermedad incurable; cuando su padre se replegaba sobre sí mismo, decían que "tenía el campo" (Friedman, 2001, p. 12).

Eso incurable, como traumatismo, es también lo que ha podido ser vivido como borradura y que consiste en haber sido negado, borrado como persona en nombre de una religión, una raza, reducido al rango de un desperdicio a eliminar.

\section{La reconciliación}

Es una cuestión siempre muy difícil de abordar y a la cual no es posible, podría decirse, responder de una manera definitiva.

La historia y la experiencia muestran - por todas partes y siempre, se podría decir en este punto - que sea cual sea el trabajo hecho - en una sociedad, un grupo, un país después de una guerra, una dictadura, una guerra civil, un tiempo de separación que los ha atravesado de una manera brutal, salvaje, etc. - , las modalidades adoptadas en cada lugar han tenido consecuencias que a menudo no se podían anticipar.

Es importante subrayar que, al lado de las decisiones de los Estados, hay también cuestiones personales de cada sujeto, que pueden encontrarse, cruzarse, separarse, oponerse...
En la Antigua Grecia encontramos un primer episodio de amnistía general. Es promulgada por Trasíbulo, vencedor de los Treinta Tiranos que, mientras gobiernan Atenas, en 404 a. C., se entregan a todos los excesos. Una vez derrotados, se les permite, con el fin de apresurar el final de la guerra, retirarse a Eleusis, una ciudad situada a unos 20 $\mathrm{km}$ de Atenas. Allí es inventada una palabra, amnêstia, que contiene a la vez la idea del perdón y la del olvido. Es de lo que se benefician y que nadie contesta; la democracia es restablecida en el país.

Posteriormente, es el tratado de paz, firmado entre Estados beligerantes, lo que regula la suerte de los participantes, mediante una amnistía general, acompañada de la imposibilidad de perseguir a cualquiera por crímenes cometidos durante los conflictos.

Con el primer conflicto mundial, se plantea la cuestión de los criminales de guerra y la posibilidad de someterlos a jurisdicciones penales nacionales e internacionales. A continuación, numerosas jurisdicciones son creadas, para — digámoslo así- no ser funcionales.

Por el contrario, los juicios de Nuremberg, de noviembre 1945 a octubre 1946, son ejemplares en cuanto al tipo de procedimiento, seguido inflexiblemente hasta su término. Además del hecho del enjuiciamiento de criminales de guerra nazis, en Nuremberg son establecidas las reglas fundamentales que rigen estos juicios. Esto sigue siendo válido hoy en día, para gran parte de los considerandos judiciales y de responsabilidad de criminales de guerra.

Demorémonos un momento en Francia y lo que ocurrió en el decurso de la Segunda Guerra Mundial. Desde junio 1944 se estableció, bajo la presidencia del General De Gaulle, el primer Gobierno Provisional de la República Francesa (GPRF) y fueron establecidas las primeras Ordenanzas que restablecieron la legalidad republicana en el país. Principalmente, preveían la creación de tribunales —Corte de Justicia y cámaras cívicas - que debían hacer pasar a la legalidad la depuración de 
la posguerra, la cual, abandonada a los ajustes de cuenta sumarios y sin juicio, ya había registrado unas 10000 ejecuciones. Esta depuración extrajudicial concitaba rencores y provocaba reacciones violentas, que algunas veces llegaban incluso a justificar a los colaboradores, a darles argumentos. En todo caso, la población francesa, fuera de los que se libraban a una venganza sanguinaria, permanecía dividida respecto a los métodos bárbaros alimentados por lo que fue llamado la leyenda negra de la depuración.

La fractura del país, al amenazar con ampliarse, llevó a De Gaulle a votar una primera ley de amnistía que excluía de condena a "los que habían obedecido órdenes y no habían tomado iniciativas". En los años siguientes, otras leyes de amnistía se extendieron a delitos o crímenes más complejos en cuanto a la implicación de las personas. Los tribunales dictaminaron un número considerable de condenas y De Gaulle, por su poder de gracia, amnistió a un gran número de ellos. Salvador de la Nación tras Londres, De Gaulle se hizo cargo, personalmente, de la necesidad imperiosa de volver a hallar una unidad.

Si bien fue seguido a nivel de la nación, eso no curó las heridas de cada uno; más aún, hubo que aprender, en lo cotidiano, a convivir con los enemigos de ayer.

Para alguien que no lo ha vivido, no se puede uno imaginar lo que representa encontrarse en los lugares públicos de un pueblo, de una pequeña ciudad, con los que han colaborado con el enemigo y le han denunciado a uno a la Gestapo o a la policía de un dictador, y que están allí disfrutando de los mismos derechos que uno, exhibiendo a veces cierta arrogancia. Entonces, y no es la menor de las paradojas, con frecuencia son los que han tenido que padecer estas violencias los que se retraen. Hasta en el cementerio continúa la proximidad, para siempre.

A las generaciones siguientes pocas cosas fueron transmitidas claramente. ¿Por qué a uno le llamaban le boche —el alemán-, despectivamente?
Probablemente su madre lo había concebido con un soldado alemán — pero, jeso no se podía decir!—. Tantas cosas hubieron de adivinar los niños, más allá de una omertà cómplice.

Digamos que eso no inclina al acercamiento ni a pensar en una paz tras la guerra. Incluso, el Estado francés tuvo que juzgar, en cuanto se acabó la guerra, a un cierto número de personas de primer plano. El caso más flagrante fue el del mariscal Pétain, que fue juzgado en 1945 por un Tribunal de Justicia superior y reconocido como de indignidad nacional; fue condenado a la pena de muerte, pena que fue conmutada por el general De Gaulle a cadena perpetua, y murió encarcelado en la isla de Yeu. Otros, ministros y funcionarios de alto rango de Vichy, fueron condenados a penas de prisión perpetua y otros fueron condenados a muerte y ejecutados (Pierre Laval, Joseph Darnand, etc.). Para otros, el pasado quedó encubierto por empleos en las altas esferas de la administración, donde mostraron su diligencia y saber hacer. Fue el caso de Maurice Papon para quien no se inició un juicio más que en 1998, por complicidad con un crimen contra la humanidad, por su responsabilidad en la deportación, desde Burdeos, de 1600 judíos, entre 1942 y 1944.

\section{Sobre el consenso}

El consenso es, al contrario de lo que piensan algunos que es para todos, lo que no consigue jamás satisfacer a nadie. El consenso es lo que hace callar, pero no quita ni el recuerdo ni el dolor. Como ofrece un trato a cada una de las partes, lo que se ha ofrecido al otro es siempre puesto en tela de juicio.

Resulta que la transición hacia la paz debe, para sostenerse políticamente, ser idealizada, para que el proceso unificador se sustente. Eso implica bastantes reajustes de lo que es la historia reciente, interpretada por unos como otros, por medio de mentiras que toman valor de verdad, de mitos que glorifican a los verdugos y otros que desvalorizan a las víctimas. 
Este proceso llevaría hacia la nivelación de los protagonistas, que es otro nombre del consenso: ¡todos habrían sufrido y habrían hecho sufrir! Es allí donde se establecería el "discurso gris": ¡nadie sería verdaderamente culpable enteramente, ni inocente del todo!; ¡todos tendrían las manos manchadas de sangre, de sangre fraterna! Entonces ¿para qué empeñarse en dar consistencia a buenos y malos, verdugos y héroes?

En esta temporalidad, la ausencia de violencia, de conflictos, la cual es necesaria para el proceso, es retomada de una manera falaz como uno de los argumentos de la ejemplaridad y de lo bien fundado de la vía elegida.

Entonces, los verdugos salen blanqueados y las víctimas son indemnizadas, según un pretium doloris, que no es más que una conminación a no seguir expresándose, puesto que la memoria de la época pasada es considerada como establecida oficialmente. Así pues, en este tiempo de transición, no se debe tampoco tocar a los símbolos que puedan evocar a un campo o al otro, siendo el argumento retorcido no borrar elementos - testimonios de la historia-, cuando de lo que se trata es más bien de no "herir susceptibilidades", no avivar nuevas divisiones. ¡Siempre se pide a las víctimas que perdonen!

El paso siguiente es "archivar" el pasado en nombre de la unidad fundamental del mañana. Una verdadera autocensura es exigida a cada uno. Luego, vendrán las leyes de amnistía donde, una vez más, es a las víctimas a quienes se pide ser clementes con sus verdugos.

Amnistía no puede ser pensada más que con amnesia y una falsa reconstrucción de la memoria histórica. Entonces el pueblo conserva sus heridas internas, que permanecen abiertas por debajo de la piel rápidamente restañada sobre heridas entumecidas. He aquí una sociedad que lleva en sí las semillas de nuevas divisiones para un futuro que no puede ir más que hacia lo peor. Pues la amnesia general o colectiva no recubrirá jamás la memoria individual y el perdón no es más que el anverso de un odio inextinguible.

La memoria es ultrajada, no por padecer amnesia, sino por haber sido alterada y oficializada tras amnistías mutuas y negociadas. Con frecuencia, demandas de perdón puramente formales, pronunciadas por los que han sostenido regímenes dictatoriales o guerra civil, son negociadas a cambio de reducciones de penas.

Aún queda apretujarse en el seno de este conjunto de fraternidad reencontrada, designando, fuera de la comunidad de pertenencia, un país, un responsable que habría empujado a lo peor.

De este modo, Joseph de Maistre (2009), en su texto Esclarecimientos sobre los sacrificios, hacía valer que "del culpable al enemigo no hubo más que un paso; todo enemigo fue culpable y aún más, desgraciadamente, todo extranjero fue enemigo, cuando hizo falta víctimas" (p. 34).

¡Lo malo está fuera, el amor ideal de la patria es reencontrado!

\section{La responsabilidad de cada uno}

Una paz duradera no puede fundarse sobre el desconocimiento de lo que ha estado en juego en las épocas revueltas, ni sobre una borradura de la memoria o la subversión del pasado.

El hombre es invariablemente empujado por una pulsión de destrucción que apunta siempre a un semejante - y no es esta la menor de las paradojas para el que no tome en cuenta lo que tanto Freud como Lacan han puesto en evidencia sobre esta cuestión-. El hombre no está jamás en paz ni con sus pulsiones, ni con sus semejantes, aunque sean los más íntimos.

Cada vez que, en nombre de la reconciliación, el pasado es vertido en el olvido público, las cicatrices de la guerra no logran cerrarse y el traumatismo jamás puede ser superado. El argumento 
de la fraternidad infiltra siempre la idea de reconciliación; pero muy precisamente lo que sabemos en psicoanálisis es que esta fraternidad está en el corazón mismo de lo que hace que los hombres se hagan la guerra.

Siempre he sostenido que, en cierto modo, todas las guerras son guerras civiles. Es siempre otro sí mismo a quien se mata en la guerra, a un hermano. Fue el grito que Henry Dunant, el fundador de la Cruz Roja, lanzó en Solferino, en el campo de la batalla espantosa que hizo en un solo día, el 24 de junio de 1859, 40000 muertos y heridos: Tutti fratelli!. Es también el tema de aquella película destacable de François Ozon, Frantz.

La guerra tiene una causa profunda que tiene que ver con la alteridad (Briole, 2015a, p. 101-106). Lacan (1984) ha insistido en la relación inicial de todo sujeto con un Otro primordial, el del "conocimiento paranoico" (p. 180) del mundo. Más allá de esta confrontación, el sujeto puede llegar a existir superando la radicalidad del “'tú o yo' permanente de una guerra, en que está en juego la existencia del uno o el otro [...]" (Lacan, 1955, p. 428). Así persiste en el inconsciente lo que engendra "la violencia del instinto de muerte freudiano, el asesinato imaginario del hermano" (Lacan, 2001b, p. 40).

No obstante, se insiste siempre en querer pensar un espacio de fraternidad donde, de nuevo, sería posible compartirlo todo - pues tanto la puesta en tela de juicio como el levantamiento del velo sobre lo que este espacio contiene hace correr el riesgo de reavivar las tensiones y abrir a nuevas guerras - Es la trampa, la elección forzada que acorrala a la víctima: ;callarse para no ser considerada como responsable del retorno de lo peor!

La idea que se impone - y que, sin embargo, sigue aún y por todas partes sin ser respetada - es la de la responsabilidad de cada uno, y sus corolarios que son las amnistías y la impunidad que, lamentablemente, vienen algunos años después.

El hombre tiene poca memoria respecto a la Historia; es olvidadizo. Mantiene una confianza en la afirmación de arrepentimiento, quiere creer en el arrepentirse del uno, en tanto que, en espejo, no puede ver en ese otro malo algo de sí mismo. El hombre está hecho a imagen del hombre, y es su drama cuando descubre que este otro verdugo que lo maltrata está hecho de la misma estofa que él. ¿Cómo en estas situaciones, poder afirmarse en tanto que hombre cuando lo que se presenta es: "soy semejante a aquel a quien, al fundarlo como hombre, fundo para reconocerme como tal" (Lacan, 1948, p. 118)?

\section{La obediencia y la responsabilidad}

En los juicios de Núremberg, el 4 de diciembre de 1945, fue adoptada la idea según la cual la obediencia militar es central en el funcionamiento de un ejército. Sin embargo, la obediencia no justifica que cualquiera cometa crímenes o actos injustificables, aunque la orden haya sido dada. "Llega un momento en que un ser humano debe rechazar obedecer a su jefe, si debe también obedecer a su conciencia. Incluso el simple soldado que sirve en las filas del ejército de su país no está obligado a obedecer órdenes ilegales". El redactado final y consensual del texto fue el siguiente: "El hecho de haber actuado bajo la orden de su gobierno o el de un superior jerárquico no libera de la responsabilidad del [agente] si ha tenido moralmente la facultad de elegir" (T.M.I Nuremberg, 1949, p. 235). He aquí planteada la facultas resistendi, esta facultad de resistir de la cual parecería que se quiere librar a los subalternos, para resarvarla al discernimiento de los decididores. Entonces, el engaño es doble: del lado decididor, no es él quien ha realizado el crimen; del lado ejecutor, no tendría verdaderamente conciencia de lo que contenían sus actos. Vercors, recordando a Macbeth, resalta que cada uno "siente sus crímenes secretos pegarse a sus manos", pero también siente que se despegan para recaer sobre el otro.

El interés superior de la nación y el patriotismo exaltado, se mezclan con el desprecio del que manda sobre los acatadores, sobre todo porque "los 
que son mandados por él obedecen al temor y no al amor". Ese jefe, el que ya no alberga amor hacia los suyos, es un "maniquí miserable" (Vercors, 2001, p. 51). El cinismo le sienta bien al que manda sobre aquellos cuya obediencia pasiva, sumisión estricta, permite que se cometan exacciones a gran escala. Ya nada se opone al horror de un goce adictivo que procura al torturador el sufrimiento y la tortura, tanto física como moral, del que es designado, entregado a la violencia mórbida del que se siente con justificación para ejercerla. Para eso no hace falta ninguna formación.

\section{La impunidad}

Sostener que la reconciliación se daría al precio de la impunidad es el error mayor que se presenta en el camino a recorrer hacia la paz.

Para una sociedad - anudamiento tan frágil de parlêtres en un mismo espacio-, la reconciliación es el precio a pagar para poner un límite al horror que se ha podido atravesar. Se trata de buscarse un porvenir común, evitando estigmatizar posiciones binarias: la radicalidad del mal para unos, la soledad del dolor de las víctimas para los otros.

El deber de memoria debe arrancarnos al olvido, pero recordarnos también que, al final de las hostilidades, se trata de ¡encubrir una impunidad, a veces castigando, para dar ejemplo! Es como si la ejecución rápida - tan estigmatizada como fuertemente mediatizada - de algunas personalidades o criminales de primer plano fuese a cubrir con un velo toda la impunidad que queda aún por despejar, uno por uno, de la masa hecha anónima.

El segundo error de lectura sería pensar que la impunidad no concierne más que a aquellos que están del lado de los vencidos (Biole, 2015b). Las exacciones en espejo cometidas por los resistentes, los oprimidos organizados en grupos de represalia, deben ser tomadas en consideración para saber lo que pueda haberlas legitimado. Se ha de decir que es una cuestión delicada y que, en
Francia, por ejemplo, jamás hubo persecuciones contra resistentes.

Otro error sería, en nombre de la constatación de que lo inhumano habría sido cometido por seres humanos, reenviar a cada uno, verdugo y víctima, espalda contra espalda, en la espiral de la violencia. Hay una impunidad legal, es la que puede establecer un Estado, y hay otra que permanece íntima para cada sujeto. A esta dimensión subjetiva, ninguna ley puede traer apaciguamiento alguno.

La evolución de la idea del deber de memoria hacia la de deber de conocimiento viene a estorbar la impunidad; no se trata únicamente de recordar, sino de avanzar hacia la verdad.

La búsqueda de la verdad, el querer saber, desplaza lo que está en juego en la impunidad. En África del Sur, el restablecimiento del diálogo entre las comunidades tras el Apartheid ha podido hacerse, en 1995, por medio de Comisiones de verdad y reconciliación. Tuvieron una importancia decisiva, por no haber retrocedido ante el hecho poder decirse lo más doloroso; ninguna de las partes rechazó aproximarse, en la medida de lo posible, a lo que había pasado.

El tiempo ni borra ni altera la memoria traumática. El tiempo judicial no coincide con el tiempo subjetivo. No hay solapamiento entre ellos. Es uno de los obstáculos con el que tropiezan muchos gobiernos que emprenden el encontrar disposiciones de pena a los que han sido reconocidos como culpables de crímenes, incluido crímenes contra la humanidad. De hecho, no hay temporalidad para la impunidad. La alternativa es la sentencia justa, la que puede pronunciar una jurisdicción de Estado y que sea aplicada según las reglas en vigor, en el momento en que han sido aceptadas como tales por las dos partes. Cada sociedad, como lo desarrolla Lacan, prevé en la "relación del crimen a la ley" un castigo. Pero, el punto decisivo, es la imperiosa necesidad de un "asentimiento subjetivo" (Lacan, 1950, p. 126) para que el castigo adquiera él mismo una significación. Este asentimiento subjetivo concierne todas las partes presentes. 
Así pues, la decisión de la Corte Suprema de Justicia de la Nación, de Argentina, del 3 de mayo de 2017, en lo que concierne a personas condenadas por crímenes contra la humanidad, respecto a la ley del 2 x 1, ha llevado a protestas encendidas, nacionales e internacionales. Esta decisión que permite a los que han sido acusados, en cuanto han cumplido dos años de detención preventiva, rebajar un día por cada día pasado en prisión, hasta que sean juzgados y hayan recibido su pena, ha sido muy mal recibida. Las víctimas han visto allí una elección por parte del Estado de una política de "desmemoria”, de olvido. Las llagas siguen allí, prestas a volver a abrirse en cuanto la clemencia hacia los verdugos adquiere la marca de lo arbitrario.

Goce desatado, locuras de guerra (Briole, 2016b).

Es un punto que he desarrollado en una Conferencia impartida hace ya un año en México, titulada El cuerpo del enemigo (Briole, 2016a), donde he subrayado cómo la guerra es este contexto en el cual lo peor del hombre puede desatarse contra su semejante. Con frecuencia, asolando y devastando el cuerpo de dicho enemigo por medio de actos bárbaros a menudo de carácter sexual. Los ejemplos son numerosos, de estos desencadenamientos incontrolables, y la lista de estos actos es infinita. El profesor Albert Bandura (1996) de la Universidad de Stanford les ha dado un nombre, casi un estatuto: moral desengagement (p. 364-374), el desenganche del sentido moral. Lo hemos considerado más bien como un tiempo de desconexión de lo simbólico, donde se hace presente "lo real como tal, el peso de lo real" (Lacan, 1959, p. 17), todo el peso de un real desatado que, sin embargo, no libra para nada el sujeto de la responsabilidad de sus actos. Estos actos, particularmente devastadores para un pueblo, no deben ser encubiertos como "hechos de guerra", sino que les corresponde su presentación ante los tribunales y un juicio que proyecte toda la luz sobre lo que ha pasado.

\section{La impunidad del soldado ordinario y la vergüenza}

En este orden ideas, pero sobre la vertiente opuesta, debemos tomar en consideración lo que adviene a los soldados que cumplen con su misión en el respeto de la Convención de Ginebra. "Los secretos guardados de uno mismo son los más pesados de llevar" — esto es lo que hace decir Kevin Powers (2013) a uno de los personajes de su libro notorio, Yellow birds, escrito tras pasar dos años en el infierno del ;Irak "liberado”!- Son tanto más pesados, estos secretos, cuanto que nadie desea escuchar lo que ellos tienen para decir. Todos los pueblos mantienen esta ambivalencia respecto a sus ejércitos. Se celebra a los militares cuando son necesarios para la protección del país, de sus intereses en el mundo, y se los estigmatiza localizando en ellos lo peor de lo que habría en el fondo de cada ser humano. Se imputa, entonces, a esos soldados el haber experimentado, en la muerte dada, un goce, tanto más malsano cuanto más marcado de impunidad; ;en la guerra se mata legalmente! Si se ha querido delegarles esta tarea, para que el país se mantenga en su lugar, y se los ha condecorado por este servicio prestado, jes para que después se callen! ¡Que resuelvan en sus casernas y consigo mismos sus estados anímicos, sin molestar a la conciencia nacional! A la falta se agrega, a veces, la vergüenza que se abate sobre ellos cuando la nación no consigue alojar la guerra que ha declarado bajo el estandarte de la moral.

Desde siempre es así: se celebran las muertes y se deja la vergüenza y la culpabilidad a los que, habiendo combatido, han salvado, sin embargo, su pellejo.

La obnubilación es tal que ni siquiera nos damos cuenta que hemos fundido en un mismo conjunto a soldados, víctimas de las guerras y deportados de los campos. A unos se le imputará goces inconfesables (masacres, torturas, violaciones, etc.) y a los otros el haber probablemente jugado su propia partida, al haber sobrevivido allí donde tantos otros 
han perecido. A todos se les pedirá que no agreguen al desorden social, político, policial, el desorden de su malestar consecutivo al encuentro traumático. Que ocupen el lugar que les está designado y no molesten a la conciencia colectiva: la del antiguo combatiente, la de la víctima.

\section{El lugar justo y la toma en consideración de las víctimas}

De todo lo que hemos podido decir hasta ahora, se notará que es delicado cernir de antemano quién será o no será considerado como víctima de un conflicto. Ocurre - y eso puede chocar - que estas víctimas pueden encontrarse en los diferentes bandos presentes. Este respeto mutuo de un sufrimiento no tiene nada de angelical, puede ser un punto de partida de una discusión abierta, donde la cuestión no es tampoco borrar los actos de cada uno.

\section{Las víctimas y las familias}

La recuperación de los cuerpos por las familias, después de su identificación, es una etapa determinante de un proceso de paz. Precisemos que este punto es igualmente importante para cada uno de los campos que han podido enfrentarse. La fosa común es una de las modalidades utilizadas en los crímenes de genocidio y las dictaduras. Lo fue también en la posguerra, durante el periodo de depuraciones. Sea del campo que sea, un cuerpo en una fosa común no está en una sepultura. Es la "desaparición de este cuerpo en la masa de los demás cuerpos" (Duras, 1994, pp. 15-56). Es su anulación en tanto que singularidad. No acceder a estas demandas legítimas agrava las tensiones, hace que la comprensión mutua sea imposible y es del orden de una cobardía política, sobre todo en la medida en que los métodos científicos actuales permiten las identificaciones.

Es una cuestión que siempre reaviva, de la manera más extrema, lo que en una nación las ha hecho posibles — recordaremos en España la trágica y escandalosa acusación de prevaricación contra el juez Baltasar Garzón en 2008 después de haber ordenado la apertura de las fosas comunes de las víctimas del franquismo (Briole, 2016a)-.

Sostenemos que no hay paz para los vivos, si no se puede realizar una paz en torno a los muertos.

La restitución de los bienes, la reintegración profesional son otros aspectos que no deben ser descuidados.

\section{El pretium doloris, el precio del dolor}

Los problemas de "reparación" a las víctimas se han planteado en cada proceso de paz, en todos los pueblos. Eso necesita estrategias delicadas, difíciles, para negociar lo mejor posible los intereses reales del sujeto. Es necesario indemnizar a una víctima, según lo que diga la ley de la nación a la cual tanto el experto como el paciente están sometidos. En determinados casos una ley debe ser pensada — adecuada al conflicto del cual se quiere salir-.

No obstante, sabemos que ningún pretium doloris puede colmar la brecha abierta en un sujeto. El reconocimiento de una víctima es también dejarle la posibilidad de hablar de lo que ha vivido y que continúa sufriendo en su fuero interior. Es necesario mucho coraje a los que han sido víctimas de esta violencia arbitraria, para llevar a cabo un trabajo sobre sí mismos, a partir de lo insólito, lo insospechado con el que se han topado y que estaba escondido en lo más profundo de su ser. Para que esto sea posible, es necesario también alguien para recibir, con tacto y atención, este sufrimiento. A veces hacen falta años para cerrar esta brecha que ha irrumpido y se ha abierto en la vida de alguien.

\section{Una escucha entre otras, la del analista}

El psicoanalista está concernido por estas demandas de ser escuchado. No debe sustraerse a ello. El psicoanalista hace lo que hace siempre: 
propone escuchar la singularidad de los efectos para cada uno del momento social, político, económico, al cual se ve confrontado. El psicoanalista no consuela, no promete mejores tiempos. Tampoco se deja instrumentalizar por los poderes públicos, para suplir su deficiencia, y ser un relevo de la insuficiencia del Estado a la hora de ocuparse de "sus" víctimas.

\section{Para una dialéctica de la paz}

No hemos considerado la reconciliación del lado del encubrimiento de las fracturas mediante un Memorial, ni del lado de las celebraciones del amor reencontrado de un pueblo dividido, ni del lado del imperativo gaullista de la necesidad urgente de "pasar página", etc. Lo hemos abordado del lado de la búsqueda de una dialéctica posible, en una sociedad que la más grande violencia ha desgarrado.

La cuestión más acuciante sigue siendo la que planteaba Lacan (1967): “¿Cómo hacer para que masas humanas, condenadas al mismo espacio, no solamente geográfico, sino eventualmente familiar, permanezcan separadas?" (p. 363). Toda guerra - de naciones, clanes, familias - desemboca en esta cuestión. Entonces, falta responder lo más cerca posible a la verdad de cada uno.

El declive de los ideales es a menudo esgrimido en el discurso contemporáneo para explicar el desencadenamiento guerrero, desordenado y multifocal, y también para justificar la subida de los racismos y de la segregación. Se finge sorpresa y desconocimiento de lo que Freud había notoriamente demostrado en su Malestar en la cultura: tras la pantalla de la civilización, que favorece la pacificación de la relación entre los hombres, nada cambia en cuanto a sus instintos fundamentales.

Las coerciones sociales, el sistema educativo, incluso el ejército, participan de una tentativa de canalizar las fuerzas instintivas, encontrándoles salidas aceptables y gestionando lo mejor posible el "resto" inherente a todo grupo social. Pero la desregulación de los lazos sociales, como la puesta en tela de juicio del Padre en su idea de Nación, reavivan las tensiones entre los grupos de un mismo Estado, hacen resurgir rencores y odios, vuelven a poner al descubierto las promesas de venganza.

El estado de derecho, el respeto de la integridad de los territorios, están y siguen estando, de hecho, aún amenazados. A pesar de que tantos combatientes, anónimos, han muerto por la libertad, "único valor imperecedero de la historia" dice Albert Camus (1951), su fragilidad es permanente y extrema. "Los hombres nunca están bien muertos más que por la libertad: no creían entonces morir del todo" (p. 299).

Pero he aquí que aún e inexorablemente, los hombres están listos para comprometerse en lo peor, tanto sienten que las redes de la guerra se estrechan en torno a ellos. Si es así, los combatientes de ayer habrán muerto una segunda vez.

El psicoanalista es también un ciudadano comprometido $y$, en este sentido, le corresponde, a partir de su formación, de su saber sobre los hombres, de su ética, decir lo que piensa y actuar en consecuencia.

\section{Referencias}

Bandura, A. (1996). Mechanisms of Moral Disengagement in the Exercise of Moral Agency. Journal of Personality and Social Psychology, 71(2), 364-374.

Briole, G. (2015a). En las fauces de la guerra: arrancamiento. En Brousse, M. El psicoanálisis a la hora de la guerra (pp. 101-106). Paris: Tres haches.

Briole, G. (2015b). El uno impune. El psicoanálisis. Revista de la Escuela Lacaniana de Psicoanálisis, 25, 76-81.

Briole, G. (2016a). El cuerpo del enemigo. Universidad del Claustro de Sor Juana. Recuperado de http://www.jornadasnel.com/Actividades-en-sedes-y-delegaciones/NEL-Mexico/16-10-14_ Conferencia-de-Guy-Briole.pdf 


\section{Guy Briole}

Briole, G. (2016b, septiembre). Impossible d'escaboter. Trabajo presentado en La psychanalyse à la lumière du gai savoir de Rabelais, Les jouissances du corps et de la parole, Colloque de Tours, Samedi.

Camus, A. (1951). L’homme révolté. Paris: Ediciones Gallimard.

De Maistre, J. (2009). Tratado sobre los sacrificios. Madrid: Sexto piso.

Duras, M. (1994). Escribir. Barcelona: Tusquets Editores.

Friedman, C. (2001). Mon père couleur de nuit. Paris: Denoël.

Lacan, J. (1946). Propos sur la causalité psychique. Paris: Seuil

Lacan, J. (1948). L'agressivité en psychanalyse Écrits. Paris: Seuil.

Lacan, J. (1950). Introduction théorique aux fonctions de la psychanalyse en criminologie Écrits. Paris: Seuil.
Lacan, J. (1955). La chose freudienne. Paris: Seuil.

Lacan, J. (1959). Le Séminaire, livre VII, L'éthique de la psychanalyse. Paris: Seuil.

Lacan, J. (1967). Allocution sur les psychoses de l'enfant. En J. Lacan, Autres écrits (pp. 361371. Paris: Seuil.

Lacan, J. (1984). Acerca de la causalidad psíquica Escritos, vol. I. Madrid: Siglo XXI.

Lacan, J. (2001a). La psychiatrie anglaise et la guerre. Autres écrits. Paris: Seuil.

Lacan, J. (2001b). Les complexes familiaux dans la formation de l'individu Autres écrits. Paris: Seuil.

Powers, K. (2013). Yellow birds. Paris: Stock.

T.M.I. Nuremberg (1949). Procès des grands criminels de guerre devant le tribunal militaire international, $t$. I. Nuremberg: T.M.I.

Tocqueville, A. (1951). Démocratie en Amérique II. Paris: Akal.

Vercors, (2001). Le silence de la mer. Paris: Magnard. 